\title{
AOCS for TanDEM-X \\ Formation flight at 200m separation in low-Earth orbit
}

\author{
J. Herman ${ }^{1}$ \\ DLR, 82234 Wessling, Germany \\ and \\ D. Fischer ${ }^{2}$ \\ EADS, 88039 Friedrichshafen, Germany \\ and \\ D. Schulze and S. Löw and M. Licht ${ }^{3}$ \\ DLR, 82234 Wessling, Germany
}

\begin{abstract}
$\mathrm{T}_{\mathrm{c}}^{\mathrm{H}}$

HE TanDEM-X project is implemented by a "Public-Private Partnership" between the German Aerospace Centre (DLR) and Astrium GmbH.

The primary goal of the TanDEM-X (TerraSAR-X add-on for Digital Elevation Measurement) mission is to generate a global digital elevation model. To achieve this, two satellites - TanDEM-X (TDX) and TerraSAR-X (TSX), a satellite of almost identical construction which is in orbit since June 2007 - will form the first configurable SAR (Synthetic Aperture Radar) interferometer in space with a separation of only a few hundred metres. A powerful ground segment, which is interlaced with that of TSX, completes the TanDEM-X system. The satellites will fly in formation and operate in parallel for three years to cover the entire surface of the Earth.

DLR is responsible for the scientific exploitation of the TSX/TDX data, as well as for planning and implementing the mission, for controlling the two satellites and for generating the digital elevation model. Astrium has built the satellites and shares in the costs of development and exploitation. As is the case already with TSX data, the responsibility for marketing the data lies in the hands of Infoterra GmbH, a subsidiary of Astrium GmbH.

AOCS operations for two spacecraft flying at low altitude with such unprecedented small separations pose several new challenges and require creative solutions, partly implemented on-board and partly on ground. Due to the extremely short reaction times, emphasis lies with the on-board handling of problems. This is achieved by implementation of a data link (one-way) between the satellites, by the regular sending of a "health" signal (twoway), by the design of a new AOCS safe mode that has no effect on the orbit, by the complete re-work of the TSX FDIR concept (on-board Fault Detection, Isolation and Recovery) and by autonomous formation control.

This paper addresses some of the more interesting on-board implementations in particular the on-board AOCS surveillance and the newly developed and implemented AOCS safe mode with the magnetic torquers as the sole actuators. It also will touch briefly on some ground aspects such as procedures, planning and execution of orbit correction manoeuvres, personnel and on-call strategy, as well as off-line analysis and tools.
\end{abstract}

\section{Nomenclature}

$\Delta v \quad=$ Velocity increment due to thruster activity

$e \quad=$ Eccentricity of the satellite's orbit

$i=$ Inclination of the satellite's orbit; both $e$ and $i$ are treated as vectors by combining the numerical value with the argument of perigee and the right ascension of the ascending node

\footnotetext{
${ }^{1}$ AOCS team lead, German Space Operations Centre, Oberpfaffenhofen, 82234 Wessling, Germany/ jaap.herman@dlr.de

${ }^{2}$ AOCS engineer, Astrium-EADS, 88039 Friedrichshafen, Germany/Denis.Fischer@astrium.eads.net

${ }^{3}$ AOCS engineers, German Space Operations Centre, Oberpfaffenhofen, 82234 Wessling,

Germany/D.Schulze@dlr.de, Sebastian.Loew@dlr.de, Maria.Licht@dlr.de
} 


\section{Introduction}

\section{A. TerraSar-X}

TerraSar-X was launched from Baikonur on June 15, 2007 in a Sun-synchronous dusk-dawn orbit at an altitude of about $515 \mathrm{~km}$. Ground coverage is repeated in an eleven day cycle, which is achieved by controlling the spacecraft in a $\pm 250 \mathrm{~m}$ tube around a prescribed Earth-fixed reference orbit (see also section III.B.).

SAR data takes are normally made under a $33.8^{\circ}$ looking angle towards the dark side of the Earth ("rightlooking"), but it is also possible to roll the spacecraft over $67.6^{\circ}$ to make a so-called "Sun-side-looking", or "leftlooking" data take. This option is used mainly over the poles where some areas would otherwise be unreachable, or when data takes are required at short notice. Images can be made in several modes with ground resolution ranging from $\sim 2 \mathrm{~m}$ to $16 \mathrm{~m}$ and an area covered from $\sim 50 \mathrm{~km}^{2}$ to $10^{4} \mathrm{~km}^{2}$. It is even possible to do interferometry by virtually splitting the SAR-antenna into two parts (dual receive mode).

The attitude in the standard operational modes is controlled within $0.3 \mathrm{mrad}(3 \sigma)$ using Astro 10 star trackers from Jena-Optronik $\mathrm{GmbH}$ and four reaction wheels from Rockwell Collins Deutschland $\mathrm{GmbH}$ with a torque capability of $0.2 \mathrm{Nm}$. Continuous wheel unloading is performed with the magnetic torque rods that are mounted parallel to the spacecraft's axes. A Mosaic GPS receiver from Astrium GmbH is used for position and velocity determination, as well as for the time synchronisation on-board.

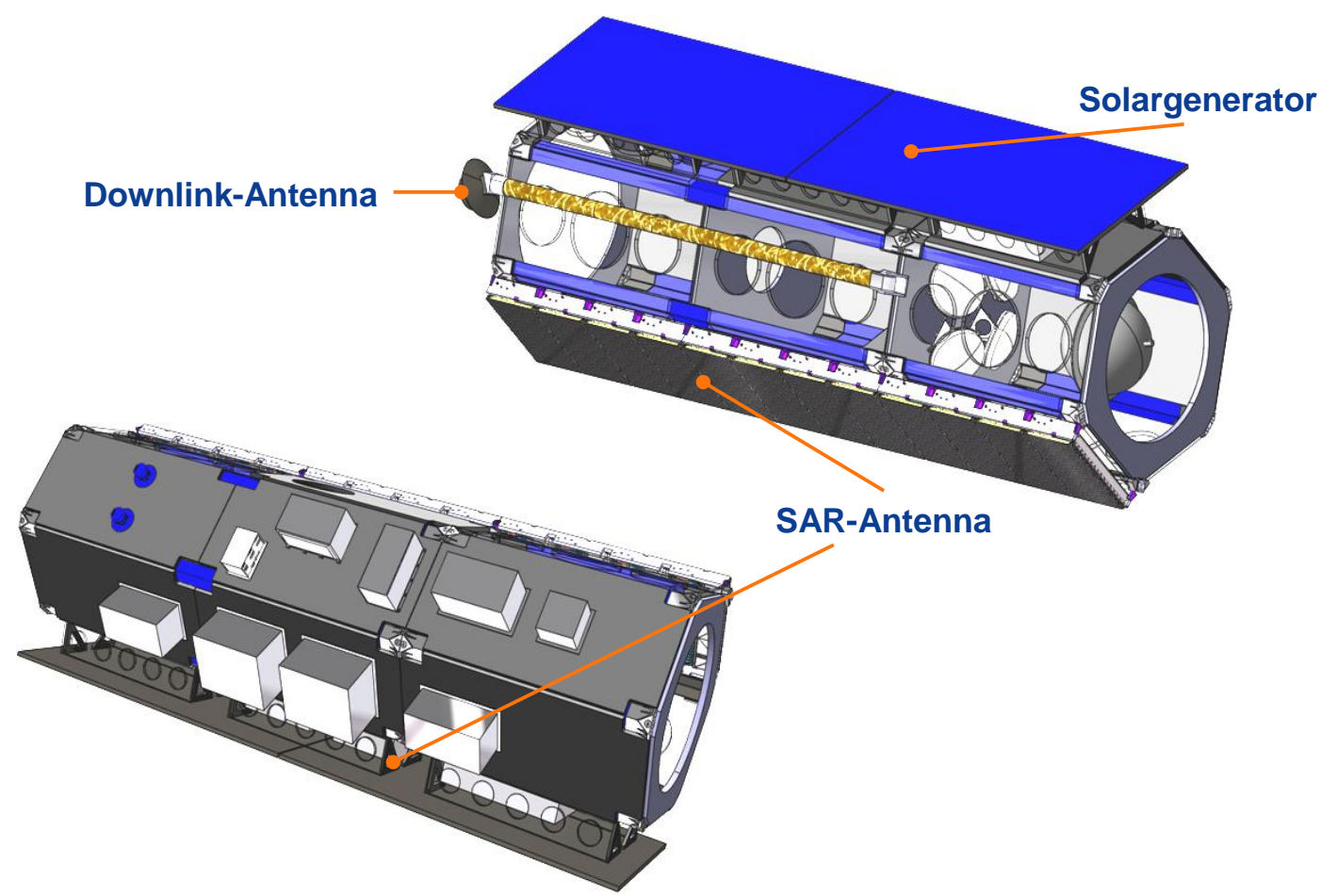

Figure 1. Schematic view of the TSX satellite. The SAR antenna consists of 12 segments of $70 \times 40$ $\mathrm{cm}^{2}$. The X-band downlink is located in the boom, which in this picture is not yet deployed. The three star trackers are mounted on the side opposite to the solar panels. The hydrazine thrusters are located at the backside and the GPS antennae on top of the spacecraft.

Orbit correction manoeuvres are made with a hydrazine propulsion system, which is pressurised by helium and operated in blow-down mode. The thrusters are arranged in two branches with four $1 \mathrm{~N}$ thrusters each that are all located at the rear of the satellite (see Fig. 2). Inclination manoeuvres, or manoeuvres in anti-flight direction thus require a $\pm 90^{\circ}$ or $180^{\circ}$ yaw slew. However, the former are made only about four times per year and the latter, which lower the orbit, occurred only during the early orbit phase directly after launch, a few times after a contingency and once when a collision with a piece of debris had to be avoided.

In case of problems in one of the operational data take-, or manoeuvre- modes the satellite is brought into a safe mode that uses measurements from the coarse Earth- and Sun-sensors (CESS), from the magnetometers and from the inertial measurement units (IMU). The hydrazine thrusters are the actuators in this mode. Each thruster has a small tilt with respect to the spacecraft's length axis, so that by selecting various pairs the three satellite axes can be controlled independently. However, attitude control with the thrusters thus produces also a force in flight direction and changes the spacecraft's orbit. A "steady-state" safe mode was therefore added, in which the 
three magnetic torque rods are the main actuators. The thrusters will still be used if the attitude errors exceed certain pre-defined limits. In this way fuel expenditure and orbital change are reduced by an order of magnitude.

\section{B. TanDEM-X}

A formation of two SAR instruments flying at cross-track separations between 200 and $2000 \mathrm{~m}$ has the advantage of a much higher image resolution. The main goal of the mission is however the creation of a global digital elevation model (DEM) with elevation accuracies better than $10 \mathrm{~m}$. The collection of DEM data will take about two years, whereby the shortest baselines have to be measured first.

Both spacecraft can also be operated in stand-alone mode during the entire mission, however.

TDX hard- and software are mainly a rebuild of TSX, but some significant changes were made to enable close formation flight. The major changes in the AOCS comprise:

- Cold gas system with 2x4 $0.04 \mathrm{~N}$ thrusters for formation-keeping manoeuvres; the thrusters are located on the spacecraft's length axis at the front- and back-sides.

- S-band receiver / decoder for TSX telemetry via inter-satellite link; 35 words with about 120 of the most important health parameters are relayed.

- Algorithms for autonomous formation flight; GPS measurements are used for orbit determination, prediction and planning of cold gas manoeuvres.

\section{Formation}

TSX will continue to be controlled on the accustomed reference orbit and TDX on a helical orbit around it. The helix is achieved by so-called $e$-,i-vector separation (relative eccentricity / relative inclination, whereby maximum cross-track separation occurs at the equator and maximum radial separation at the poles; see Ref. 4). Both spacecraft will carry out the standard hydrazine manoeuvres with the same $\Delta v$ (see section III.B.1.). Additionally, formation keeping- or reconfiguration- manoeuvres will be made by TDX alone with the cold gas system ${ }^{*}$.

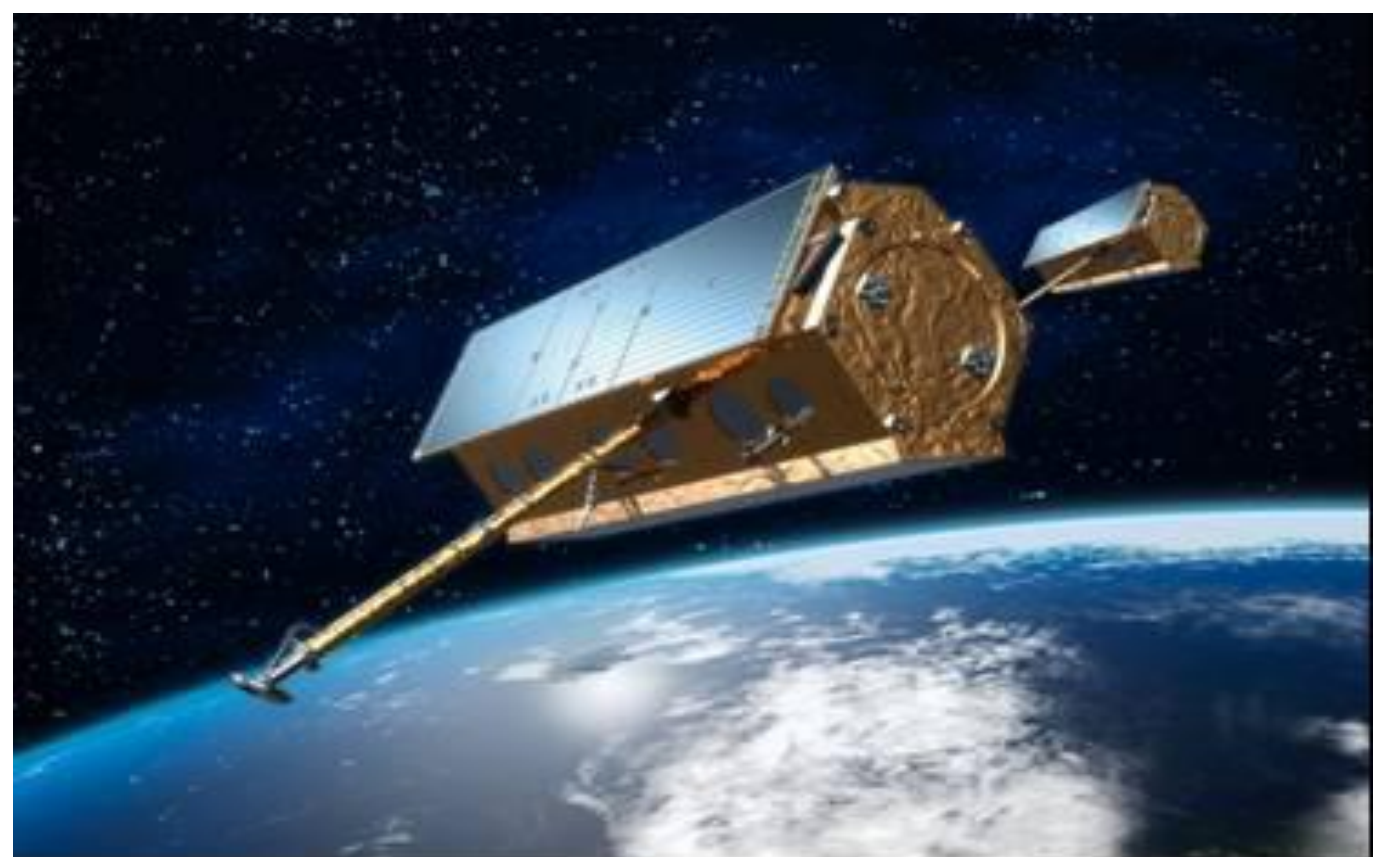

Figure 2. TSX and TDX in close formation. The SAR antenna is below and the solar panels on the left hand side. The $2 \times 4$ hydrazine thrusters at the rear of the satellite are well visible.

Two spacecraft of more than $1300 \mathrm{~kg}$ each, flying with $7 \mathrm{~km} / \mathrm{s}$ at a separation of $200 \mathrm{~m}$ are an operational challenge to ground- and space- segment. The major risks are:

- Instrument damage by radiation; a direct hit of the SAR signal, which delivers pulses of $\mathrm{kW}$ strength, in the instrument of the second satellite would be up to nine orders of magnitude stronger than the normally detected back-scatter from the Earth's surface, which is of the order of $\mu \mathrm{W}$.

\footnotetext{
* In fact any manoeuvre type on TDX with a $\Delta v>1 \mathrm{~cm} / \mathrm{s}$ will invoke the hydrazine thrusters and any manoeuvre $<1 \mathrm{~cm} / \mathrm{s}$ will be made with the cold gas system; in praxis this corresponds to the stated segregation
} 
- Collision; uncontrolled changes of the orbit can lead to a considerable increase of the collision risk.

Both points are primarily guarded against by avoiding uncontrolled orbit changes. The implementation in the AOCS will be subject of section III. Radiation damage is also avoided by prohibiting data takes when the second satellite is passing through the beam of the first. These so-called "exclusion zones" depend upon the operational mode and both "right-looking" and "left-looking" must be accounted for. The duration for crossing the exclusion zone depends upon inter-satellite distance and constellation.

\section{Inter-satellite communication}

\section{A. Inter-satellite link}

The existing S-band downlink system on TSX is used to relay important health data to a dedicated receiver / decoder added on TDX. Note that the traffic is one-way, because a corresponding receiver is not available on TSX.

The inter-satellite link (ISL) has some limitations. First of all, it only works when the TSX transmitter is in low-rate $(32 \mathrm{kbit} / \mathrm{s})$, which normally coincides with low-power. This limits the operational distance to $1.5-2 \mathrm{~km}$ (under special circumstances low-rate/high-power could be used, which would allow connection over distances of $\left.5-9 \mathrm{~km}^{1}\right)$. The TSX transmitter is normally switched to high-rate $(1 \mathrm{Mbit} / \mathrm{s})$ and high-power for each station contact, which implies a regular interruption of the ISL with gaps of typically $10-20 \mathrm{~min}$. Secondly, the patchand helix-antennae point for the normal spacecraft orientation towards zenith and nadir respectively. This leads to some distance degradation and short dead zones when the spacecraft are horizontally aligned.

\section{B. Sync pulses}

Bi-static operations, where the SAR signals of the one spacecraft are also detected by the other, require synchronous processing of both data takes. The synchronous start of data takes is achieved by exchanging "sync pulses" based upon the GPS sync signal at a rate of $\sim 5 \mathrm{~Hz}$ (configurable).

Sync pulses are transmitted and received by six horns, which are mounted in such a way that $4 \pi$ sr coverage is achieved. Best signal-to-noise ratio is obtained by selecting the appropriate pair.

\section{Sync warning data takes}

Sync warning data takes are normal, albeit very short, data takes where the signal is sent out by one satellite and detected by the second. Again a selected pair of sync horns is used for this operation and not the SAR instrument. A positive result will allow SAR data takes on the receiving side for the next 50 minutes.

The aim of regular sync warning data takes is to detect any problem - e.g. a drop to AOCS safe mode - on the sending satellite in which case instrument operations on the receiving spacecraft will immediately be suspended.

\section{Safety measures}

\section{A. Exclusion zones}

Data takes on one of the satellites are only allowed if the other spacecraft is not within the beam of the outgoing SAR signal. The half-power beam width is about $30^{\circ}$ and directed either to the dark, or the illuminated side of the Earth. An illustration is given in Fig. 3, where the formation is seen along the flight trajectory.

Only data takes that do not violate the constraints can be loaded to the spacecraft and carried out. Exclusion zone checks are first made on ground and will prevent commands to be uploaded in case conflicts are detected. FDIR surveillance on-board provides a final safety check.

Note that the exclusion zones are enlarged (to $\sim 45^{\circ}$ in Fig. 3 ) after orbit maintenance manoeuvres in order to safeguard against unexpected performance errors. 


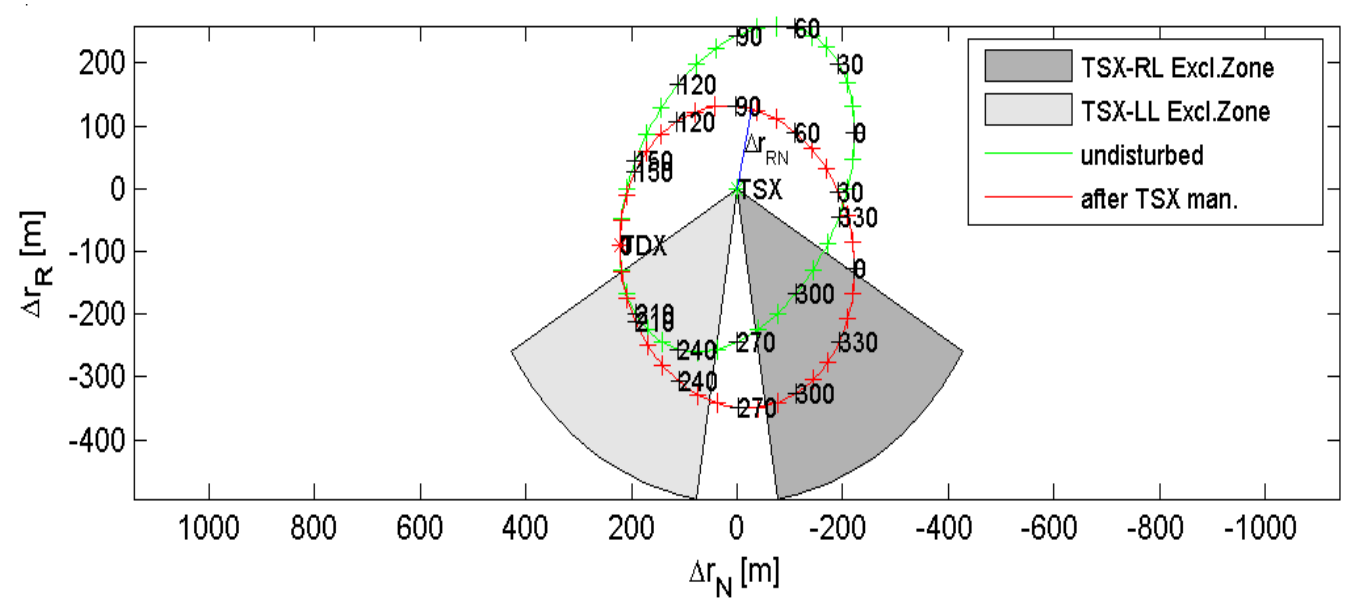

Figure 3. An example is shown of TDX flying through the exclusion zones of TSX. View is along the flight trajectory with TSX fixed at the centre. The grey shaded areas show the exclusion zones for the "right-looking" and "left-looking" modes of TSX. The green line is the relative and undisturbed orbit of TDX leading in this example to an exclusion zone of $\pm 30^{\circ}$ (indicated by the tick marks). An orbit manoeuvre on TSX only would lead to the relative trajectory shown by the red line resulting in $a \pm 45^{\circ}$ exclusion zone (see Ref. 5). Minimum cross-track separation after the manoeuvre is shown by the blue vector $\Delta r_{R N}$.

\section{B. Manoeuvre planning}

\section{Simultaneous orbit corrections (hydrazine)}

The standard orbit correction manoeuvres to maintain the TSX reference orbit (see section I.A.) will be executed simultaneously by TDX. These manoeuvres are made with the hydrazine thrusters and comprise roughly four inclination manoeuvres per year of $\leq 30 \mathrm{~cm} / \mathrm{s}$ and in-plane burns of $\sim 1 \mathrm{~cm} / \mathrm{s}$ every 10 to 14 days. The strength, duration and frequency of the in-plane manoeuvres depend upon tank pressure and upon solar activity (actually the height of the remnant atmosphere). It is expected that this will increase to some $5 \mathrm{~cm} / \mathrm{s}$ every second day, if the solar maximum 2013 becomes comparable to the ones in 1991 and 2002.

The planning of the manoeuvres will be such that no enhanced risk of radiation damage or collision will ensue, even if one or both of the burns is not or only partially carried out. Large manoeuvres might consequently be split into two parts.

\section{Formation keeping (cold gas)}

All formation keeping manoeuvres will be made on TDX alone. Larger corrections, e.g. to correct an inclination difference, use the $1 \mathrm{~N}$ hydrazine thrusters, but the majority of the formation keeping will be done with the $40 \mathrm{mN}$ thrusters of the cold gas system.

Safety measures comprise a limitation of the number of manoeuvres per orbit and a limit to the duration of $300 \mathrm{~s}$, which yields a maximum $\Delta v$ that can not threaten the formation.

Passive safety is given by the fact that the formation is stable for at least a week $(9.5$ days are actually expected) without any manoeuvres at all. This also safeguards against failed, or only partially carried out cold gas manoeuvres.

\section{TAFF: TanDEM autonomous formation flight}

The software on TDX contains a module for the on-board computation of the relative orbit. The TAFF module, standing for TanDEM autonomous formation flight, has three distinct operational modes:

i) navigation only:

Relative orbit determination based upon GPS navigation data is done on-board, as well as the orbit prediction (e.g. to bridge gaps in the ISL). Planning, realisation and commanding of formation keeping manoeuvres is done on ground.

ii) open loop: In addition to the above, the in-plane formation keeping manoeuvres will also be computed on-board. Realisation and commanding is still done by ground, however. 
iii) closed loop:

Full on-board autonomy, including commanding of all in-plane cold gas manoeuvres. The only remaining ground activity is a posteriori verification, calibration and the planning of the out-of-plane manoeuvres.

Intensive ground support still remains a necessity even when operating in "closed loop". In addition to the above mentioned points, also the target parameters for the formation have to be reset regularly and sent to the spacecraft. Sets of manoeuvre and other control parameters, as well as planned TSX orbit corrections also have to be uploaded routinely for the TAFF to operate.

\section{Safe mode modification}

The original safe mode concept on TSX was based upon CESS, magnetometer and IMU as sensors and the $1 \mathrm{~N}$ hydrazine thrusters as actuators. Analyses in preparation of close formation flight showed that the orbit changes in case of a drop to the thruster based safe mode would lead to a $10-100$ times higher collision risk.

It was therefore decided to implement a second safe mode branch on both satellites with the magnetic torque rods as the sole actuators. The torque rods and magnetometers are operated in alternation to allow disturbance free measurements of the Earth's magnetic field.

The concept was tested on TSX in flight and proved to be feasible and robust, thus presenting the possibility to avoid orbital changes completely in case of an AOCS safe mode entry. A few provisos remain, however (see also section III.D.):

- The alternate usage of the magnetic torque rods and the magnetometers limits its use to rotation rates $<2 \%$. This is four times larger than all rates that are commanded on-board, yet there might be contingencies where higher rotation rates occur.

- The initial rotation rates might indeed have been $<2 \%$, but it could happen that even after three orbits they still remain $>0.14 \%$ implying that the board-autonomous transition to Earth- and Sun-acquisition mode (ASM-MTQ-AQ) did not yet take place.

- Control authority of the magnetic torquers is relatively low with a maximum moment of $110 \mathrm{Am}^{2}$. Recovery can take several orbits which might lead to a power problem, or to unacceptably low or high temperatures on some prime components.

In such cases a switch to the thruster based safe mode must be made as ultima ratio, yet in first instance with a limitation on the total $\Delta v$. This limit depends on the actual formation settings and will be chosen such that the resulting maximum change in the orbit does not substantially increase the collision risk.

\section{FDIR concept}

The FDIR concept was completely reworked in preparation for close formation flight. The major changes with respect to the original TSX implementation are:

- The additional magnetorquer safe mode (ASM-MTQ; functionality, rotation rate, two step approach).

- Thruster safe mode (ASM-RCS; surveillance of accumulated thruster on-time, two step approach).

- Surveillance of the ISL, TAFF, sync pulses and sync warning data takes.

- System-level reactions, comprising safety measures in several sub-systems in parallel.

- Additional power/thermal monitoring functions.

The logic for the handling of the safe mode is shown in Fig. 4. Entry point in case of problems in one of the operational modes (NOM is the nominal operation mode; OCM is the orbit control mode) will always be ASMMTQ-RD (rate damping in magnetorquer safe mode). A board-autonomous sequence will bring the spacecraft in Earth/Sun acquisition mode (ASM-MTQ-AQ) once the rotation rates are below a certain threshold that is currently set at $0.14 \%$. If the rates are not below the specified limit after $\sim 3$ orbits, an on-board computer (OBC) warm boot is triggered. Thereafter the spacecraft starts again in ASM-MTQ-RD. The thruster safe mode will only be invoked after the $5^{\text {th }} \mathrm{OBC}$ reboot. Exception to this scenario occurs in case of severe thermal, or power problems in which case the transition into ASM-RD occurs immediately.

The total amount of $\Delta v^{\dagger}$ upon first entry of ASM-RCS is limited, such that the maximum orbit change does not lead to a substantially increased collision risk ${ }^{\ddagger}$. The actual limits depend upon inter-satellite distance and current tank pressure. Once the $\Delta v$ limit is reached an $\mathrm{OBC}$ reboot is triggered, which returns the spacecraft to ASM-MTQ-RD.

Unlimited thruster activity can only occur at second ASM-RCS entry.

\footnotetext{
In fact the equivalent thruster on-time

${ }^{\ddagger}$ FDIR reaction inhibits data takes on both satellites in case of a mode drop on one, thus eliminating the risk of radiation damage
} 


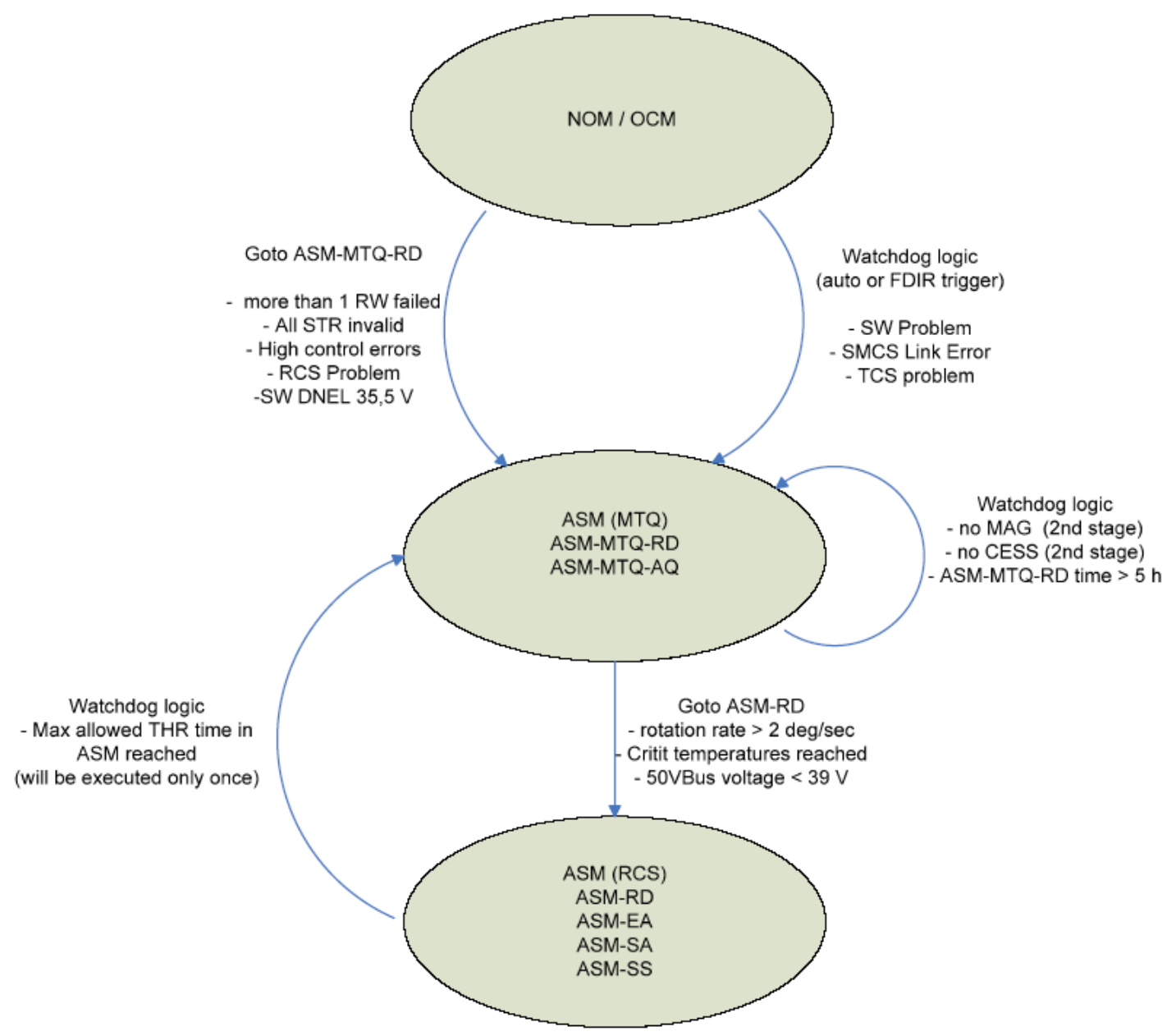

Figure 4. Modified FDIR scheme developed in preparation for close formation flight, which is implemented on TSX already. The nominal operational modes are on top of the diagram, followed by the magnetic $-M T Q-$ and thruster $-R C S-$ safe modes. The sub-modes refer to rate damping $(R D)$, Earth- or Sun-acquisition (AQ, EA, SA) and steady state (SS). The several paths in the logic are indicated together with the triggering events (see Ref. 2, their figure 8.2-7)

Some cases remain that could still lead to uncontrolled orbital changes due to unlimited thruster activity in safe mode:

- high rotation rates of at least $2 \%$ s; e.g.

a reaction wheel gets stuck (speed should go to zero in safe mode)

a mechanical open failure on one of the hydrazine thrusters

a hit by another object

- failure of selected critical thermistors (e.g. on solar panels, star trackers, or flow control valves)

- main bus voltage too low $(<39 \mathrm{~V})$

- OBC reboot with EEPROM settings (after four boots were made with safe guard memory); e.g. both magnetometers failed (also after rebooting)

CESS persistent failure

MTQ-RD timer triggering the $5^{\text {th }}$ boot

OBC problems leading to more than 4 resets

None of these cases has a high probability and none of these cases has ever been observed in flight on TSX.

\section{E. Station coverage}

The number of ground stations per day and the interval between contacts depend upon the FDIR concept (see section III.D.). The current implementation normally guarantees at least $2 \times 3$ orbits, or roughly ten hours in total during which no orbital changes will occur, when the satellite drops to safe mode. 
Contacts are therefore separated by at most six hours. This allows the detection of a safe mode in the most unfavourable case six hours after it occurred on-board. Offline data can then be analysed in order to find the cause of the problem and to prepare measures to mitigate it. This, together with ordering additional station support or calling people in outside working hours, will take $\leq 2$ orbits, thus keeping the reaction time below ten hours in total.

\section{F. On-call strategy and automatic data surveillance}

The traditional on-call strategy at GSOC had one engineer reachable by telephone outside working hours with round-the-clock attendance for station contacts and routine operations provided by a contracted multimission team. This approach guaranteed the safety of the spacecraft, but repairs in case of problems in specific areas - e.g. instrument, flight dynamics, or AOCS - had to wait for the first normal working day, unless of course the responsible expert happened to be the person on-call.

The need for quicker reaction in formation flight, also on sub-system level, coupled with the wish to minimise interruption of payload operations, led to a considerably extended concept which is in force already for TSX (see Ref. 3). On-call service is provided simultaneously for the following areas:

- Mission planning and operation (supplemented by the mission manager in case of really critical contingencies)

- $\quad$ AOCS

- $\mathrm{OBC}$

- Power/thermal

- $\quad$ Flight dynamics

- $\quad$ SAR instrument

- $\quad$ LCT, the laser communication terminal (not for TDX)

Engineers are still reachable by telephone, but also have laptops equipped with wireless internet access. This allows immediate monitoring of live telemetry, writing or authorising of recommendations in order to send commands to the spacecraft, and access to E-mail traffic, spacecraft manuals and documentation.

Finally, a large number of critical parameters in each sub-system is automatically screened and compared with pre-defined limits in the live telemetry and in the dump data. Limit violations lead to warnings (yellow flags) or alarms (red flags) in the live telemetry and generate an E-mail notification when they occur in the dump data. The E-mail lists in detail which parameters in a given dump file are out-of-limit, the severity of the violation and the number of occurrences. Full details, such as time and measured value, are provided in an attached Excel sheet.

\section{Conclusions}

All preparations for the formation flight of TSX and TDX are finished. The new AOCS safe mode using the magnetic torque rods as sole actuators has been implemented and was successfully tested in orbit. The new FDIR concept and the extended on-call strategy are also fully operational after an extensive test phase. The algorithms for the computation of the orbit and formation correction manoeuvres on TDX are running in parallel with the TSX planning for more than half a year already.

Unfortunately, the launch of TDX was delayed by 6-12 months due to a potential risk on some electronic components within the instrument. It is now scheduled for late May 2010 with the first close formation of $200 \mathrm{~m}$ separation about three months later.

\section{Acknowledgments}

The authors wish to thank R. Kahle, A. Spörl and U. Steinbrecher for their helpful comments and critical reading of the text. 


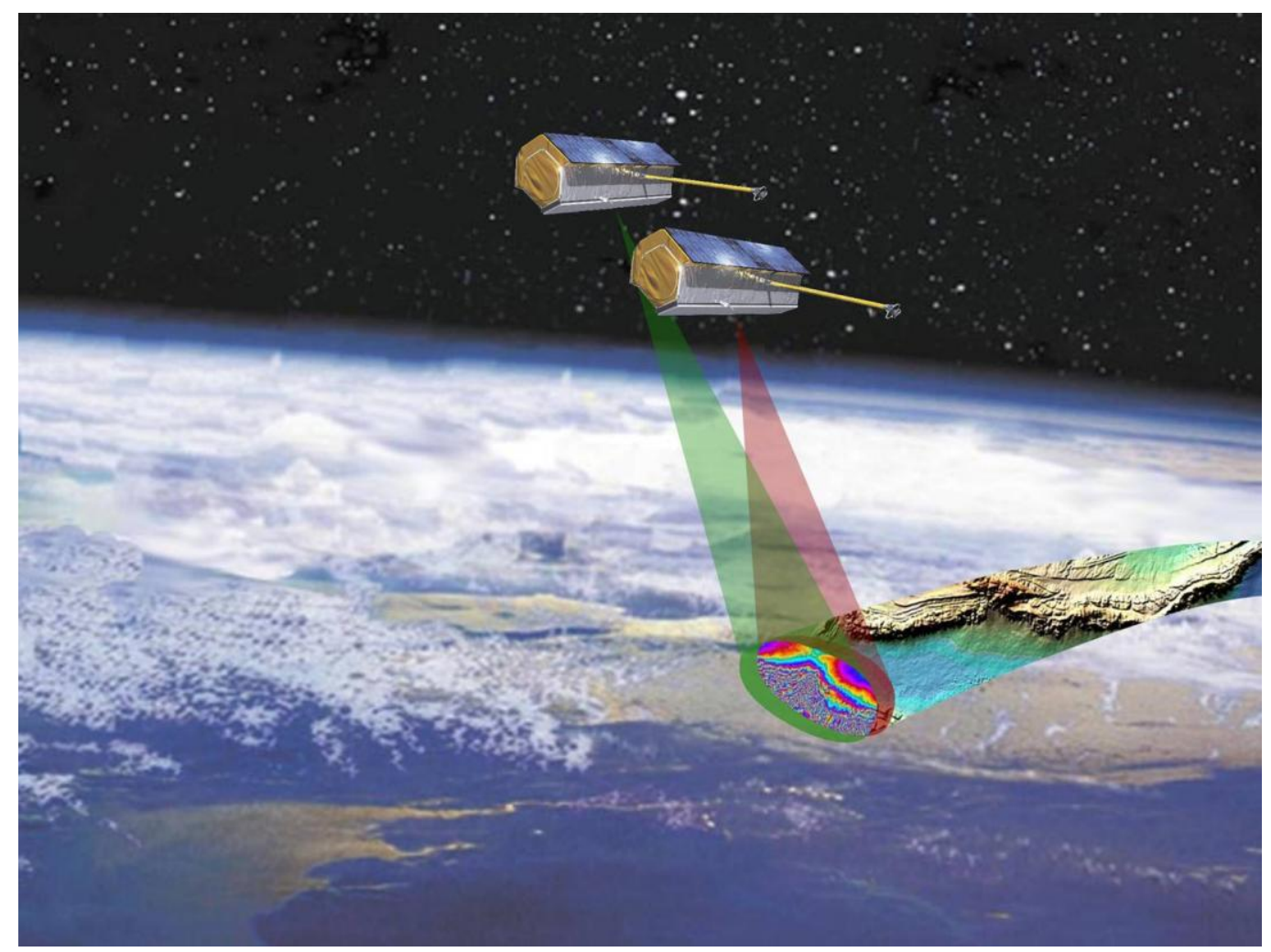

Figure 5. Impression of the near future. One of the satellites sends radar pulses illuminating a swath, or a hot spot on the Earth's surface. The back-scatter is detected by both SAR antennae enabling the construction of a digital elevation model. The orientation of the satellites indicates that in this case a "left-looking" data take is made. Note that pulses can only be sent if the second spacecraft is not in the exclusion zone. In a special application pulses are sent alternating from each spacecraft and received by both. No other configurations will be routinely used, apart from the stand-alone mode.

\section{References}

1 TanDEM-X; Inter-Satellite Link - Decoder; TDX-AED-DD-0002; Astrium, 2007

2 TanDEM-X: Design \& Interface Document; TDX-AED-DD-0001; Astrium 2008

3 Codazzi, A.; "Extended On-Call Support for TerraSAR-X; SpaceOps conference; Huntsville, Alabama 2010

4 D'Amico, S.,Montenbruck, O.: Proximity Operations of Formation-Flying Spacecraft using eccentricity/inclination vector separation, Journal of Guidance, Control, and Dynamics, 2005

5 Kahle, R.; TSX/TDX Formation Collision \& Illumination Aspects; TD-MOS-TN-4060; GSOC 2008 